\title{
Oferta y demanda regional de carne de pollo en México, 1996-2016
}

Eulogio Rebollar Rebollar ${ }^{\text {a }}$

Alfredo Rebollar Rebollar ${ }^{b}$

Jaime Mondragón Ancelmo a

Germán Gómez Tenorio ${ }^{\mathrm{a}^{*}}$

${ }^{a}$ Universidad Autónoma del Estado de México. Centro Universitario UAEM Temascaltepec. Km. 67.5, carretera Toluca-Tejupilco. Colonia Barrio de Santiago s/n. 51300 Temascaltpec. Estado de México, México.

${ }^{\mathrm{b}}$ Universidad Tecnológica del Sur del Estado de México. Estado de México, México.

* Autor de correspondencia: gomte61@ yahoo.com

\section{Resumen:}

El objetivo de la investigación fue determinar la magnitud del efecto de las principales variables económicas y tecnológicas que influyen en la oferta y demanda de carne de pollo en ocho regiones de México, durante el periodo de 1996 a 2016. Se formuló un modelo econométrico de regresión lineal múltiple para cada región, donde se incluyeron las variables económicas y tecnológicas principales que determinan la oferta y demanda. La oferta carne de pollo en canal reacciona directa y elásticamente a cambios en la tecnología, directa e inelásticamente por el precio de la carne de pollo e inversa e inelásticamente por el precio de la carne de cerdo y precio del alimento, en la mayoría de las regiones, cuyos valores promedio regionales fueron 1.7395, 0.9912, -.03686 y 0.1423. La demanda se comportó de manera elástica, respecto al tamaño de la población e inelástica en relación al precio corriente de la carne de pollo, ingreso per cápita y precio corriente de la carne de bovino en todas las regiones, reportando valores promedio de $2.0853,-0.1698,0.2560$ y 0.0272 ; el crecimiento de la población fue la variable que mayor incidencia reportó sobre el consumo de carne de pollo en las distintas regiones de México. Todos los modelos tuvieron significancia global; sin embargo, no todas las variables predictoras mostraron significancia individual. 
Palabras clave: Carne de pollo, Elasticidades, Producción, Consumo, Modelos econométricos.

Recibido:03/04/2018

Aceptado: 13/07/2018

\section{Introducción}

En México, la avicultura es la actividad más dinámica de la ganadería nacional, debido al crecimiento en la producción y consumo, además de un grado mayor de integración en comparación con los otros sectores pecuarios ${ }^{(1)}$, siendo la principal industria trasformadora de proteína vegetal en proteína animal ${ }^{(2)}$; lo cual confirma la posición de la carne de pollo como la proteína preferida de los consumidores mexicanos ${ }^{(3)}$.

Durante 1996 a 2016, la producción nacional de carne de pollo registró una tasa de crecimiento medio anual (TCMA) de $4.55 \%$. En 1996, la producción fue 1.26 millones de toneladas, mientras que, en 2016, esta cifra ascendió a 3.07 millones. En este periodo, el consumo nacional aparente, pasó de 1.39 a 3.84 millones, representando $5.21 \%$ de incremento medio anual; lo cual significó un crecimiento mayor comparado con la dinámica de la producción nacional. Esta diferencia de volúmenes, fue cubierta por importaciones, las cuales, aumentaron en promedio anual $7.66 \%$ y representaron alrededor de $18 \%$ del consumo de este producto ${ }^{(3)}$.

El dinamismo de la producción avícola al interior del país evidenció disparidades entre las regiones a través del tiempo. En 2016, las regiones Centro Occidente (CO) y Centro-Este (CE), lograron un fuerte dinamismo económico, ya que en conjunto aportaron el $53.37 \%$ de la producción nacional; las regiones Noreste (NE) y Península de Yucatán (PE) aportaron apenas el $7.76 \%{ }^{(4)}$.

En este periodo, el precio de la carne de pollo en canal se comportó de manera heterogénea entre regiones. En 2016, la región CO, principal productora de carne de pollo, reportó un precio de 30.53 pesos por kilogramo $(\$ / \mathrm{kg})$; inferior en $3.38 \%$ en relación al año anterior; debido a las mejoras en las condiciones de producción y control de la gripe aviar; adicional al decremento de los precios de los principales granos forrajeros en el mercado internacional; lo anterior contribuyó a una disminución de los costos de producción de esta especie pecuaria ${ }^{(5)}$. En la región CE el precio fue de 29.68 \$ $/ \mathrm{kg}$; mientras que la región PE tuvo un precio de $32.22 \$ / \mathrm{kg}$ y en la Oriente el precio fue de $33.37 \$ / \mathrm{kg}^{(4)}$.

Por otra parte, el sorgo como principal componente del alimento para pollo ${ }^{(6)}$ también reportó variaciones en distintas zonas del país. Así durante 2016, en las regiones Noreste, Norte y Centro 
Este, reportó incrementos porcentuales de 10.13, 8.41, $2.84 \%$; en tanto que, en la zona, Península de Yucatán el precio disminuyó $2.84 \%$, respecto a $2015^{(4)}$.

En relación a la demanda, de 1996 a 2016, el consumo regional de carne de pollo en las zonas Centro Este, Centro Occidente y Sur, obtuvieron una TCMA de 5.15, 4.95 y $4.98 \%$, este comportamiento se explicó en parte por el incremento en el producto interno bruto (PIB) per cápita de $2.67,4.35$ y $3.27 \%{ }^{(7)}$, el crecimiento de la población a nivel regional 1.17, 0.99 y $1.02^{(8)}$, así como por la preferencia del consumidor, número de integrantes en la familia e ingreso que afectan positivamente la probabilidad de consumo de carne ${ }^{(9)}$.

Las diferencias en la dinámica de las diferentes variables económicas y tecnológicas que determinan la oferta y demanda de carne de pollo en el ámbito regional son evidentes; por ello, es importante representar mediante modelos econométricos el funcionamiento de los diferentes mercados regionales; a fin de generar herramientas que contribuyan a orientar a los hacedores de políticas públicas y dispongan de alternativas para el diseño de programas de apoyo a esta actividad, desde una perspectiva territorial.

Por lo anterior, el objetivo de la investigación fue determinar la magnitud del efecto de las principales variables económicas y tecnológicas que influyen en la oferta y demanda de carne de pollo en ocho regiones de México (Noroeste, Norte, Noreste, Centro Occidente, Centro Este, Sur, Oriente y Península de Yucatán) durante el periodo de 1996 a 2016. La hipótesis considera que existen diferencias en la magnitud de las variables que influyen en la oferta y demanda de carne de pollo en las distintas regiones de México.

\section{Material y métodos}

La regionalización, es una metodología, procedimiento o intervención para modificar el orden territorial de un país en unidades territoriales más pequeñas, con características comunes, y representa una herramienta metodológica básica en la planeación ambiental, ya que permite el conocimiento de los recursos para su manejo adecuado ${ }^{(10)}$. Por tanto, para analizar el comportamiento de la oferta y demanda de carne de pollo a nivel regional, México se dividió en ocho regiones económicas $^{(11)}$ (Cuadro 1). 
Cuadro 1: Regiones y entidades de México

\begin{tabular}{ll}
\hline Región & Entidades \\
\hline Noroeste (NO) & Baja California, Baja California Sur, Sonora, Sinaloa y Nayarit \\
Norte (NR) & Chihuahua, Coahuila, Durango San Luis Potosí y Zacatecas \\
Noreste (NE) & Nuevo León y Tamaulipas \\
Centro-Occidente (CO) & Aguascalientes, Colima, Guanajuato, Jalisco y Michoacán \\
Centro-Este (CE) & $\begin{array}{l}\text { Ciudad de México, Hidalgo, Estado de México, Morelos, } \\
\text { Puebla, Querétaro y Tlaxcala }\end{array}$ \\
Sur (SU) & Chiapas, Guerrero y Oaxaca \\
Oriente (OR) & Tabasco y Veracruz \\
\hline
\end{tabular}

Se formuló un modelo econométrico de regresión lineal múltiple para la oferta y demanda de carne de pollo en canal en cada región de México, durante el periodo de 1996 a 2016. La oferta incluyó las variables: precio de la carne de pollo, la tecnología (medida como eficiencia alimenticia) y el precio de los insumos (alimento) ${ }^{(12)}$. La demanda, consideró el precio de la carne de pollo, ingreso monetario, población y los precios de los productos sustitutos o complementarios ${ }^{(13)}$. Los modelos representan el comportamiento interno de los diferentes mercados regionales.

La información correspondiente a cada una de las variables, se obtuvo de fuentes como SIAP (Servicio de Información Agroalimentaria y Pesquera), FIRA (Fideicomisos Instituidos en Relación con la Agricultura), INEGI (Instituto Nacional de Estadística y Geografía), CONAPO (Consejo Nacional de Población) y SNIIM (Sistema Nacional de Información e Integración de Mercados). La eficiencia alimenticia se obtuvo de $\operatorname{Rigolin}^{(14)}$ (varios años), sin embargo, como este indicador se reportó cada 10 años; el resto de los años se estimaron a través de la fórmula del crecimiento promedio anual, definida por $r \mathrm{r}=\left(\mathrm{D}_{\mathrm{f}} / \mathrm{D}_{\mathrm{i}}\right)^{1 / \mathrm{n}}-1$; donde $\mathrm{D}_{\mathrm{f}}$ y $\mathrm{D}_{\mathrm{i}}$ son el dato final e inicial de la eficiencia alimenticia y, la $\mathrm{r}$ la tasa de crecimiento media anual.

Para estimar el valor de los parámetros en los modelos lineales asociados a la función de oferta y demanda, se utilizó el método de Mínimos Cuadrados Ordinarios (MCO $)^{(15)}$, con el paquete estadístico $\mathrm{SAS}^{(16)}$, que permitió conocer el efecto de cada una de las variables independientes sobre la dependiente, además de obtener los mejores estimadores lineales insesgados y de varianza mínima.

La congruencia estadística de los modelos de oferta y demanda, se determinó por medio del coeficiente de determinación $\left(\mathrm{R}^{2}\right)$, la significancia estadística, de cada ecuación se efectuó mediante la prueba F, y la significancia individual de cada coeficiente con la t de Student o la "razón de t". La evaluación económica, se realizó al considerar los signos y la magnitud de los 
coeficientes de las variables de las funciones de oferta y demanda, y se interpretaron de acuerdo con los fundamentos de la teoría económica; es decir, la relación entre la oferta y el precio de la carne de pollo en canal, así como con la eficiencia alimenticia, debe ser directa; mientras que con el precio de la carne de cerdo y precio del alimento inversa; por su parte, la relación entre la demanda y el precio corriente de la carne de pollo en canal, debe ser inversa; y respecto al PIB per cápita, precio corriente de la carne de bovino y población humana, directa.

En algunas regiones, las variables, precio de la carne de pollo, de la carne de cerdo (bien alternativo) y precio del alimento se rezagaron un año o dos años, debido a que el productor no reacciona de inmediato a un cambio en los precios disminuyendo la producción y, factores como la duración del ciclo productivo de los animales, el grado de inversión realizada, el volumen de producción y la situación financiera de la empresa, son diferentes en las distintas regiones.

Asimismo, se determinaron las elasticidades económicas de cada una de las variables explicativas que afectaron la oferta y demanda de carne de pollo en cada una de las regiones y, se evaluaron de acuerdo al signo y magnitud de sus coeficientes; los cuales, también se interpretaron acorde a la teoría económica.

La forma estadística de los modelos econométricos propuestos para determinar los factores que determinan la oferta y demanda en los distintos mercados regionales de carne de pollo son:

$\mathrm{OCP}_{\mathrm{t}}=\beta_{11}+\beta_{12} \mathrm{PCPR}_{\mathrm{t}}+\beta_{13} \mathrm{PCCR}_{\mathrm{t}-2}+\beta_{14} \mathrm{PAR}_{\mathrm{t}}+\beta_{15} \mathrm{EA}_{\mathrm{t}}+\mathfrak{f}_{\mathrm{t}}$ (Noroeste y Norte)

$\mathrm{OCP}_{\mathrm{t}}=\beta_{21}+\beta_{22} \mathrm{PCPR}_{\mathrm{t}}+\beta_{23} \mathrm{PCCR}_{\mathrm{t}}+\beta_{24} \mathrm{PAR}_{\mathrm{t}}+\beta_{25} \mathrm{EA}_{\mathrm{t}}+\mathfrak{L}_{\mathrm{t}}$ (Noreste, Centro Occidente y Centro Este)

$\mathrm{OCP}_{\mathrm{t}}=\beta_{31}+\beta_{32} \mathrm{PCPR}_{\mathrm{t}-1}+\beta_{33} \mathrm{PCCR}_{\mathrm{t}-2}+\beta_{34} \mathrm{PAR}_{\mathrm{t}}+\beta_{35} \mathrm{EA}_{\mathrm{t}+} \mathfrak{f}_{\mathrm{t}}$ (Sur y Oriente)

$\mathrm{OCP}_{\mathrm{t}}=\beta_{41}+\beta_{42} \mathrm{PCPR}_{\mathrm{t}}+\beta_{43} \mathrm{PCCR}_{\mathrm{t}-2}+\beta_{44} \mathrm{PAR}_{\mathrm{t}-2}+\beta_{45} \mathrm{EA}_{\mathrm{t}+} \mathfrak{£}_{\mathrm{t}}($ Península de Yucatán)

$\mathrm{DCP}_{\mathrm{t}}=\beta_{51}+\beta_{52} \mathrm{PCPR}_{\mathrm{t}}+\beta_{53} \mathrm{PIBRP}_{\mathrm{t}}+\beta_{54} \mathrm{PRCB}_{\mathrm{t}}+\beta_{55} \mathrm{POB}_{\mathrm{t}}+\mathfrak{f}_{\mathrm{t}}$

Donde:

$\mathrm{OCP}_{\mathrm{t}}$ : cantidad ofertada de carne de pollo en canal en el periodo actual, aproximada a la producción regional de este producto $(\mathrm{t})$;

PCPR $_{\mathrm{t}}$ : precio real promedio ponderado regional de la carne de pollo en canal, en el periodo actual $(\$ / \mathrm{kg})$

PCPRt 1 : precio real promedio ponderado regional de la carne de pollo en canal, con un año de rezago $(\$ / \mathrm{kg})$;

$\mathrm{PCCR}_{\mathrm{t}}$ : precio real promedio ponderado regional de la carne de cerdo, en periodo actual $(\$ / \mathrm{kg})$; PCCR $_{\mathrm{t}-2}$ : precio real promedio ponderado regional de la carne de cerdo, con dos años de rezago, como producto alterativo $(\$ / \mathrm{kg})$;

PAR $_{\mathrm{t}}$ : precio real promedio ponderado regional del alimento para pollo, en el periodo actual, aproximado por el precio del sorgo como ingrediente principal $(\$ / \mathrm{kg})$; 
PARt-2: precio real promedio ponderado regional del alimento para pollo, con dos años de rezago, aproximado por el precio del sorgo como ingrediente principal $(\$ / \mathrm{kg})$;

$\mathrm{EA}_{\mathrm{t}}$ eficiencia alimenticia;

$\mathrm{DCP}_{\mathrm{t}}$ : volumen de demanda de carne de pollo en canal en el periodo actual, aproximado por el consumo aparente regional (miles de toneladas);

PCPR $_{\mathrm{t}}$ : precio real promedio ponderado regional de carne de pollo en canal en el periodo actual $(\$ / \mathrm{kg})$;

PIBRP $_{t}$ : producto interno bruto real per cápita regional en el periodo actual (miles de \$/persona), como una variable de aproximación al ingreso nacional disponible per cápita; $\mathrm{PRCB}_{\mathrm{t}}$ : precio real promedio ponderado regional de la carne de bovino $(\$ / \mathrm{kg})$, como producto sustituto;

$\mathrm{POB}_{\mathrm{t}}$ : población regional en el periodo actual (millones de habitantes/región).

Todas las variables monetarias se deflactaron con el Índice Nacional al Consumidor (INPC, base $2012=100)$.

La formulación de los modelos está basada en la teoría económica y evidencia empírica. Se ha encontrado que los productores de carne de pollo en México fundan sus decisiones de aumentar, mantener o disminuir su producción, dependiendo del precio del pollo, de los precios de los insumos necesarios para producirlo y de los bienes alternativos como el cerdo ${ }^{(17,18)}$.

Para reflejar el progreso tecnológico en el volumen de producción de carne de pollo se utilizó la EA, ya que es una de las variables que más influyen en la producción del sector avícola, y un factor que ha estimulado el crecimiento de la producción de carne de pollo en las diferentes regiones de México, misma que mediante la selección genética ha originado un pollo que produce mayor cantidad de carne con la misma cantidad de alimento; lo anterior, ha propiciado que el avicultor continúe ofreciendo su producto, apoyado en el aumento de la productividad de las aves ${ }^{(6)}$.

La eficiencia alimenticia, es una variable que integra avances tecnológicos y permite explicar por qué el avicultor continúa ofreciendo su producto en el mercado, a pesar de una clara tendencia a la baja en el precio de la carne de pollo, adicional a incrementos en el precio del sorgo como principal insumo alimenticio ${ }^{(6)}$. Otra variable que se incluyó fue el precio de la carne de cerdo como un bien alternativo, debido a que algunas empresas ${ }^{(19,20,21)}$, producen pollo y cerdo y, utilizan los mismos insumos para su alimentación ${ }^{(17,22)}$.

Los precios de la carne de pollo y cerdo se determinaron a través del precio real promedio ponderado regional del producto en canal; el precio del alimento, se consideró el precio del sorgo por ser el principal componente para la elaboración de este producto ${ }^{(6)}$. Todos los precios se obtuvieron del promedio ponderado de todas las entidades que integran cada una de las regiones de México.

La demanda regional de carne de pollo, se determinó al considerar el consumo aparente por región como una variable de aproximación a la demanda regional de este producto; este consumo regional, 
se estimó por la producción, más importaciones, menos exportaciones, a nivel regional; luego, se multiplicó por el número de habitantes por región del país del año determinado de la serie de tiempo considerada.

Con base en la teoría económica las variables determinantes de la demanda que se incluyeron en el modelo fueron: el precio real promedio ponderado regional de la carne de pollo en canal $\left(\mathrm{PCPR}_{\mathrm{t}}\right)$, ingreso (producto interno bruto real per cápita regional $\mathrm{PIBRP}_{\mathrm{t}}$ ), precio del bien sustituto (precio real promedio ponderado regional de la carne de bovino $\left(\mathrm{PRCB}_{\mathrm{t}}\right)$ y la población regional $\left(\mathrm{POB}_{\mathrm{t}}\right.$ ), todas las variables en el periodo actual ${ }^{(12,13)}$.

Para determinar el valor de las elasticidades por región en relación a cada una de las variables explicativas, se multiplicaron los coeficientes de las derivadas parciales de las ecuaciones regionales, por el valor final observado de cada una de las variables independientes respecto de las cantidades ofrecidas y demandadas. Dado que las funciones lineales de oferta y demanda tienen una elasticidad variable a través de su rango de estimación, se obtuvo para el último año del periodo analizado, por ser cercano a la actualidad ${ }^{(23)}$; así, se cuantificaron los efectos establecidos en las relaciones funcionales.

\section{Resultados y discusión}

Los resultados estadísticos obtenidos a partir de los modelos de oferta y demanda estimados en su forma lineal en cada una de las regiones de México, se presentan en los Cuadros 2 y 3. Las ecuaciones de oferta de carne de pollo en canal, en la mayoría de las regiones presentaron un coeficiente de determinación considerado como alto, el valor mayor lo tuvo la región $\mathrm{CO}\left(\mathrm{R}^{2} 98\right)$, y el menor la región $\mathrm{NE}\left(\mathrm{R}^{2} 65\right)$. En relación a los modelos de demanda, los coeficientes de determinación en las regiones fueron de 0.98 y 0.99. Las zonas de Noroeste, Norte, Noreste, Centro Este y Península de Yucatán fueron las que mejor se ajustaron a los datos. De acuerdo a la prueba de hipótesis realizada con el estadístico F de Fisher para determinar la significancia global del modelo de oferta y demanda, fue significativo al $95 \%(P<0.05)$.

Cuadro 2: Coeficientes estimados para la oferta regional de carne de pollo en canal en México, 1996-2016

\begin{tabular}{lllllllll}
\hline Región & $\begin{array}{l}\text { Variable } \\
\text { dependiente }\end{array}$ & Intercepto & \multicolumn{2}{l}{ Variables explicativas } & & \multirow{2}{*}{$\mathbf{R}^{\mathbf{2}}$} & \multicolumn{2}{c}{ Prob>F } \\
\hline $\mathrm{NO}$ & $\mathrm{OCP}_{\mathrm{t}}$ & & $\mathrm{PCPR}_{\mathrm{t}}$ & $\mathrm{PCCR}_{\mathrm{t}-2}$ & $\mathrm{PAR}_{\mathrm{t}}$ & $\mathrm{EA}_{\mathrm{t}}$ & 0.97 & 0.0001 \\
& Coeficiente & -283.530 & 0.018 & -0.084 & -3.023 & 0.696 & &
\end{tabular}




\begin{tabular}{|c|c|c|c|c|c|c|c|c|}
\hline & Error estándar & 80.766 & 7.594 & 1.474 & 4.555 & 0.193 & & \\
\hline & Valor de $\mathrm{t}$ & -3.511 & 0.002 & -0.057 & -0.664 & 3.602 & & \\
\hline \multirow[t]{4}{*}{ NR } & $\mathrm{OCP}_{\mathrm{t}}$ & & $\mathrm{PCPR}_{\mathrm{t}}$ & PCCR $_{\mathrm{t}-2}$ & $\mathrm{PAR}_{\mathrm{t}}$ & $\mathrm{EA}_{\mathrm{t}}$ & 0.97 & 0.0001 \\
\hline & Coeficiente & -429.039 & 5.467 & -2.241 & -41.770 & 1.315 & & \\
\hline & Error estándar & 119.379 & 16.808 & 2.725 & 14.331 & 0.482 & & \\
\hline & Valor de $\mathrm{t}$ & -3.594 & 0.325 & -0.823 & -2.915 & 2.728 & & \\
\hline \multirow[t]{4}{*}{$\mathrm{NE}$} & $\mathrm{OCP}_{\mathrm{t}}$ & & $\mathrm{PCPR}_{\mathrm{t}}$ & $\mathrm{PCCR}_{\mathrm{t}}$ & $\mathrm{PAR}_{\mathrm{t}}$ & $\mathrm{EA}_{\mathrm{t}}$ & 0.65 & 0.0024 \\
\hline & Coeficiente & -498.555 & 17.329 & -4.062 & -15.540 & 0.559 & & \\
\hline & Error estándar & 374.659 & 9.015 & 1.230 & 5.862 & 0.255 & & \\
\hline & Valor de $\mathrm{t}$ & -1.331 & 1.922 & -3.301 & -2.651 & 2.191 & & \\
\hline \multirow[t]{4}{*}{$\mathrm{CO}$} & $\mathrm{OCP}_{\mathrm{t}}$ & & $\mathrm{PCPR}_{\mathrm{t}}$ & $\mathrm{PCCR}_{\mathrm{t}}$ & $\mathrm{PAR}_{\mathrm{t}}$ & $\mathrm{EA}_{\mathrm{t}}$ & 0.98 & 0.0001 \\
\hline & Coeficiente & -859.623 & 25.465 & -1.955 & -2.452 & 1.449 & & \\
\hline & Error estándar & 219.764 & 18.368 & 2.105 & 15.680 & 0.475 & & \\
\hline & Valor de $\mathrm{t}$ & -3.912 & 1.386 & -0.929 & -0.156 & 3.054 & & \\
\hline \multirow[t]{4}{*}{$\mathrm{CE}$} & $\mathrm{OCP}_{\mathrm{t}}$ & & $\mathrm{PCPR}_{\mathrm{t}}$ & $\mathrm{PCCR}_{\mathrm{t}}$ & $\mathrm{PAR}_{\mathrm{t}}$ & $\mathrm{EA}_{\mathrm{t}}$ & & \\
\hline & Coeficiente & -174.121 & 36.242 & -3.750 & -34.724 & 0.026 & 0.96 & 0.0001 \\
\hline & Error estándar & 173.069 & 11.621 & 1.192 & 10.502 & 0.252 & & \\
\hline & Valor de $\mathrm{t}$ & -1.006 & 3.119 & -3.146 & -3.306 & 0.102 & & \\
\hline \multirow[t]{4}{*}{ SUR } & $\mathrm{OCP}_{\mathrm{t}}$ & & $\mathrm{PCPR}_{\mathrm{t}-1}$ & $\mathrm{PCCR}_{\mathrm{t}-2}$ & $\mathrm{PAR}_{\mathrm{t}}$ & $\mathrm{EA}_{\mathrm{t}}$ & 0.97 & 0.0001 \\
\hline & Coeficiente & -255.419 & 0.595 & -0.142 & -3.699 & 0.598 & & \\
\hline & Error estándar & 56.058 & 7.348 & 0.911 & 8.832 & 0.303 & & \\
\hline & Valor de t & -4.556 & 0.081 & -0.156 & -0.419 & 1.975 & & \\
\hline \multirow[t]{4}{*}{ OR } & $\mathrm{OCP}_{\mathrm{t}}$ & & PCPR $_{\mathrm{t}-1}$ & PCCR $_{\mathrm{t}-2}$ & PAR $_{t}$ & $\mathrm{EA}_{t}$ & 0.89 & 0.0001 \\
\hline & Coeficiente & -69.288 & 1.798 & -5.019 & -11.709 & 0.759 & & \\
\hline & Error estándar & 144.104 & 10.379 & 3.787 & 13.598 & 0.501 & & \\
\hline & Valor de $\mathrm{t}$ & -0.481 & 0.173 & -1.325 & -0.861 & 1.515 & & \\
\hline \multirow[t]{4}{*}{ PE } & $\mathrm{OCP}_{\mathrm{t}}$ & & $\mathrm{PCPR}_{\mathrm{t}}$ & $\mathrm{PCCR}_{\mathrm{t}-2}$ & PAR $_{t-2}$ & $\mathrm{EA}_{\mathrm{t}}$ & & \\
\hline & Coeficiente & -44.205 & 8.873 & -2.421 & -0.218 & 0.019 & 0.94 & 0.0001 \\
\hline & Error estándar & 18.465 & 5.491 & 0.644 & 3.957 & 0.210 & & \\
\hline & Valor de $\mathrm{t}$ & -2.394 & 1.616 & -3.759 & -0.055 & 0.090 & & \\
\hline
\end{tabular}

$\mathrm{OCP}_{\mathrm{t}}=$ cantidad ofertada de carne de pollo en canal en el periodo actual; $\mathrm{PCPR}_{\mathrm{t}}=$ precio real promedio ponderado regional de la carne de pollo en canal, en el periodo actual; PCPRt - $_{\text {= }}$ precio real promedio ponderado regional de la carne de pollo en canal, con un año de rezago; $\mathrm{PCCR}_{\mathrm{t}}=$ precio real promedio ponderado regional de la carne de cerdo, en periodo actual; $\mathrm{PCCR}_{\mathrm{t}-2}$ = precio real promedio ponderado regional de la carne de cerdo, con dos años de rezago; $\mathrm{PAR}_{\mathrm{t}}=$ precio real promedio ponderado regional del alimento para pollo, en el periodo actual; $\mathrm{PARt}_{-2}=$ precio real promedio ponderado regional del alimento para pollo, con dos años de rezago; $\mathrm{EA}_{\mathrm{t}}=$ eficiencia alimenticia. 
Cuadro 3: Coeficientes estimados para la demanda regional de carne de pollo en canal en México, 1996-2016

\begin{tabular}{|c|c|c|c|c|c|c|c|c|}
\hline \multirow{2}{*}{$\begin{array}{l}\text { Región } \\
\mathrm{NO}\end{array}$} & \multirow{2}{*}{$\begin{array}{l}\begin{array}{l}\text { Variable } \\
\text { dependiente }\end{array} \\
\mathrm{DCP}_{\mathrm{t}}\end{array}$} & \multirow[t]{2}{*}{ Intercepto } & \multicolumn{4}{|c|}{ Variables explicativas } & \multirow{2}{*}{$\begin{array}{l}\mathbf{R}^{\mathbf{2}} \\
0.99\end{array}$} & \multirow{2}{*}{$\begin{array}{c}\text { Prob>F } \\
0.0001\end{array}$} \\
\hline & & & $\mathrm{PCPR}_{\mathrm{t}}$ & PIBRP $_{t}$ & $\mathrm{PRCB}_{\mathrm{t}}$ & $\mathrm{POB}_{\mathrm{t}}$ & & \\
\hline & Coeficiente & -323.601 & -2.783 & 0.192 & 0.424 & 61.174 & & \\
\hline & Error estándar & 14.927 & 0.842 & 0.183 & 0.296 & 3.615 & & \\
\hline & Valor de $\mathrm{t}$ & -21.679 & -3.304 & 1.045 & 1.433 & 16.923 & & \\
\hline \multirow[t]{4}{*}{ NR } & $\mathrm{DCP}_{\mathrm{t}}$ & & $\mathrm{PCPR}_{\mathrm{t}}$ & PIBRP $_{t}$ & $\mathrm{PRCB}_{\mathrm{t}}$ & $\mathrm{POB}_{\mathrm{t}}$ & 0.99 & 0.0001 \\
\hline & Coeficiente & -496.889 & -3.278 & 0.826 & 0.350 & 66.127 & & \\
\hline & Error estándar & 64.500 & 0.835 & 0.423 & 0.373 & 9.670 & & \\
\hline & Valor de $\mathrm{t}$ & -7.704 & -3.926 & 1.955 & 0.937 & 6.838 & & \\
\hline \multirow[t]{4}{*}{$\mathrm{NE}$} & $\mathrm{DCP}_{\mathrm{t}}$ & & $\mathrm{PCPR}_{\mathrm{t}}$ & $\operatorname{PIBRP}_{t}$ & $\mathrm{PRCB}_{\mathrm{t}}$ & $\mathrm{POB}_{\mathrm{t}}$ & 0.99 & 0.0001 \\
\hline & Coeficiente & -251.052 & -1.161 & 0.304 & 0.353 & 54.096 & & \\
\hline & Error estándar & 13.831 & 0.358 & 0.072 & 0.125 & 2.934 & & \\
\hline & Valor de $\mathrm{t}$ & -18.152 & -3.247 & 4.250 & 2.828 & 18.440 & & \\
\hline \multirow[t]{4}{*}{$\mathrm{CO}$} & $\mathrm{DCP}_{\mathrm{t}}$ & & $\mathrm{PCPR}_{\mathrm{t}}$ & PIBRP $_{t}$ & $\mathrm{PRCB}_{\mathrm{t}}$ & $\mathrm{POB}_{\mathrm{t}}$ & 0.98 & 0.0001 \\
\hline & Coeficiente & -722.606 & -7.026 & 1.872 & 0.071 & 64.197 & & \\
\hline & Error estándar & 114.992 & 2.109 & 0.516 & 0.536 & 10.298 & & \\
\hline & Valor de $\mathrm{t}$ & -6.284 & -3.331 & 3.629 & 0.133 & 6.234 & & \\
\hline \multirow[t]{4}{*}{$\mathrm{CE}$} & $\mathrm{DCP}_{\mathrm{t}}$ & & $\mathrm{PCPR}_{\mathrm{t}}$ & PIBRP $_{t}$ & $\mathrm{PRCB}_{\mathrm{t}}$ & $\mathrm{POB}_{\mathrm{t}}$ & & \\
\hline & Coeficiente & -1538.963 & -5.887 & 4.901 & 0.297 & 55.572 & 0.99 & 0.0001 \\
\hline & Error estándar & 136.262 & 3.515 & 1.223 & 1.486 & 8.610 & & \\
\hline & Valor de $\mathrm{t}$ & -11.294 & -1.675 & 4.009 & 0.200 & 6.455 & & \\
\hline \multirow[t]{4}{*}{ SUR } & $\mathrm{DCP}_{\mathrm{t}}$ & & $\mathrm{PCPR}_{\mathrm{t}}$ & PIBRP $_{t}$ & $\mathrm{PRCB}_{\mathrm{t}}$ & $\mathrm{POB}_{\mathrm{t}}$ & 0.98 & 0.0001 \\
\hline & Coeficiente & -614.336 & -1.525 & 1.906 & 0.001 & 72.960 & & \\
\hline & Error estándar & 60.190 & 0.702 & 0.506 & 0.453 & 7.604 & & \\
\hline & Valor de $\mathrm{t}$ & -10.207 & -2.174 & 3.765 & 0.001 & 9.595 & & \\
\hline \multirow[t]{4}{*}{ OR } & $\mathrm{DCP}_{\mathrm{t}}$ & & $\mathrm{PCPR}_{\mathrm{t}}$ & PIBRP $_{t}$ & $\mathrm{PRCB}_{\mathrm{t}}$ & $\mathrm{POB}_{\mathrm{t}}$ & 0.98 & 0.0001 \\
\hline & Coeficiente & -671.124 & -3.195 & 0.430 & 0.011 & 98.815 & & \\
\hline & Error estándar & 64.780 & 0.475 & 0.119 & 0.331 & 8.462 & & \\
\hline & Valor de $\mathrm{t}$ & -10.360 & -6.729 & 3.610 & 0.033 & 11.678 & & \\
\hline \multirow[t]{4}{*}{$\mathrm{PE}$} & $\mathrm{DCP}_{\mathrm{t}}$ & & $\mathrm{PCPR}_{\mathrm{t}}$ & PIBRP $_{t}$ & $\mathrm{PRCB}_{\mathrm{t}}$ & $\mathrm{POB}_{\mathrm{t}}$ & & \\
\hline & Coeficiente & -96.599 & -0.444 & 0.102 & 0.000 & 49.720 & 0.99 & 0.0001 \\
\hline & Error estándar & 20.881 & 0.133 & 0.099 & 0.050 & 8.560 & & \\
\hline & Valor de $\mathrm{t}$ & -4.626 & -3.332 & 1.024 & 0.003 & 5.808 & & \\
\hline
\end{tabular}

$\mathrm{DCP}_{\mathrm{t}}=$ demanda de carne de pollo en canal en el periodo actual; $\mathrm{PCPR}_{\mathrm{t}=}$ precio real promedio ponderado regional de carne de pollo en canal en el periodo actual; $\mathrm{PIBRP}_{\mathrm{t}}$ producto interno bruto real per cápita regional en el periodo actual; $\mathrm{PRCB}_{\mathrm{t}}=$ precio real promedio ponderado regional de la carne de bovino; $\mathrm{POB}_{\mathrm{t}}=$ población regional en el periodo actual. 
La contribución de cada una de las variables explicativas en ambos modelos se evaluaron de acuerdo a su t asintótica o razón de t, la cual debe ser mayor que la unidad, situación que indica que el valor del parámetro estimado, es mayor que su error estándar ${ }^{(24)}$. Bajo este argumento, por el lado de la oferta, el coeficiente de las variables eficiencia alimenticia resultó significativo en la mayoría de las regiones, con excepción de CE y PE; por otro lado, el coeficiente de las variables precio de la carne de pollo, fue significativo en cuatro regiones $\mathrm{NE}, \mathrm{CO}, \mathrm{CE}$ y $\mathrm{PE}$ mientras que NO, NR, SUR y OR, no cumplieron con el parámetro establecido.

El coeficiente de la variable precio de la carne de cerdo, fue significativo en las regiones NE, CE, OR y PE; en tanto que las regiones NO, NR, CO y SUR no cumplieron con la medida. En los modelos de demanda, los coeficientes precio de la carne de pollo, producto interno bruto per cápita y población resultaron significativos $(P<0.05)$, para todas las regiones; la variable precio de la carne de bovino, fue significativo en las regiones $\mathrm{NO}$ y NE, el resto de las regiones no cumplieron con la condición antes señalada. Los resultados obtenidos indicaron que no hubo multicolinealidad entre las variables explicativas en los modelos regionales de oferta y demanda, usando la regla práctica de Klein ${ }^{(25)}$.

\section{Elasticidades regionales}

Para medir la magnitud de la variable dependiente ante variaciones, (ceteris paribus) con cada una de las explicativas de los modelos de oferta y demanda, se determinaron los coeficientes de las elasticidades por región (Cuadro 4). Las magnitudes de las elasticidades de oferta y demanda en relación a cada una de las variables independientes fueron distintas en cada una de las regiones de México; es decir, el efecto que éstas producen sobre la producción de carne de pollo, fue diverso entre los territorios. En relación a la oferta de este producto pecuario en la mayoría de las regiones, se explica directa y elásticamente por la tecnología (EA), con excepción de las regiones CE y PE cuya relación fue directa pero inelástica; directa e inelásticamente por el precio de la carne de pollo en las regiones NO, NR, CO, SUR y OR, y directa y elástica en las regiones NE, CE y PE; inversa e inelásticamente por el precio de la carne de cerdo y precio del alimento excepto NE que fue inversa y elástica para la carne de cerdo. 
Cuadro 4: Elasticidades regiones de oferta y demanda de carne de pollo en canal en México, 1996- 2016

\begin{tabular}{lllllllll}
\hline & \multicolumn{7}{c}{ Regiones } \\
\cline { 2 - 9 } Elasticidad & NO & NR & NE & CO & CE & SUR & OR & PE \\
\hline Oferta & & & & & & & & \\
PCPR $_{t}$ & 0.0021 & 0.2824 & 3.6332 & 0.8125 & 1.5794 & - & - & 1.7069 \\
PCPR $_{\mathrm{t}-1}$ & - & - & - & - & - & 0.0941 & 0.1616 & - \\
PCCR $_{\mathrm{t}}$ & - & - & -1.4563 & -0.0913 & -0.2024 & - & - & - \\
PCCR $_{\mathrm{t}-2}$ & -0.0110 & -0.1615 & - & - & - & -0.0253 & -0.4738 & -0.5273 \\
PAR $_{\mathrm{t}}$ & -0.0325 & -0.1899 & -0.4256 & -0.0084 & -0.1436 & -0.0475 & -0.0876 & - \\
PAR $_{\mathrm{t}-2}$ & - & - & - & - & - & - & - & -0.0034 \\
EA $_{\mathrm{t}}$ & 2.2151 & 1.8561 & 4.6830 & 1.2548 & 0.0299 & 2.2015 & 1.5884 & 0.0872 \\
& & & & & & & & \\
Demanda & & & & & & & & \\
PCPRt $_{\text {PIBRPt }}$ & -0.2101 & -0.2127 & -0.1037 & -0.2767 & -0.1196 & -0.1014 & -0.2525 & -0.0819 \\
PRCBt & 0.0741 & 0.2844 & 0.2159 & 0.3557 & 0.5457 & 0.2763 & 0.1428 & 0.1536 \\
POBt & 0.0656 & 0.0506 & 0.0817 & 0.0063 & 0.0122 & 0.0001 & 0.0017 & 0.0001 \\
\hline
\end{tabular}

$\mathrm{PCPR}_{\mathrm{t}}=$ precio real promedio ponderado regional de la carne de pollo en canal, en el periodo actual; PCPRt-1 = precio real promedio ponderado regional de la carne de pollo en canal, con un año de rezago; $\mathrm{PCCR}_{\mathrm{t}}=$ precio real promedio ponderado regional de la carne de cerdo, en periodo actual; $\mathrm{PCCR}_{\mathrm{t}-2}=$ precio real promedio ponderado regional de la carne de cerdo, con dos años de rezago; $\mathrm{PAR}_{\mathrm{t}}$ = precio real promedio ponderado regional del alimento para pollo, en el periodo actual; PARt - $_{2}$ precio real promedio ponderado regional del alimento para pollo, con dos años de rezago; $\mathrm{EA}_{\mathrm{t}}=$ Eficiencia alimenticia. $\mathrm{PCPR}_{\mathrm{t}}$ precio real promedio ponderado regional de carne de pollo en canal en el periodo actual; $\mathrm{PIBRP}_{\mathrm{t}}$ Producto interno bruto real per cápita regional en el periodo actual; $\mathrm{PRCB}_{\mathrm{t}}=$ precio real promedio ponderado regional de la carne de bovino; $\mathrm{POB}_{\mathrm{t}}=$ población regional en el periodo actual.

Los incrementos que se registraron en el volumen de la producción carne de pollo en la mayoría de las regiones se debieron al cambio tecnológico, definido como eficiencia alimenticia, ya que esta variable registró las elasticidades mayores en relación al resto, acentuándose en los territorios NE, NO y SUR, cuyos valores fueron 4.683, 2.215 y 2.201 respectivamente. Por su parte, las regiones $\mathrm{NR}$, OR y CO se manifestaron de manera menos elástica $(1.856,1.588,1.255)$ que las anteriores, mientras que las regiones $\mathrm{CE}$ y $\mathrm{PE}$ obtuvieron elasticidades por debajo de la unidad (0.030, 0.087), por lo que su comportamiento fue inelástico en relación al cambio tecnológico. Estas discrepancias en las elasticidades son debidas a los diferentes precios de los insumos y de la carne de pollo entre las regiones.

La tecnificación de la actividad avícola reduce costos de producción y mejora la productividad ${ }^{(6)}$; Por tanto, ante un incremento del $10 \%$ en esta variable tecnológica, ocasionaría que la curva de oferta de carne de pollo en canal se desplace a la derecha y, la producción de esta especie en las regiones NE, NO y SUR, se aumentaría en 46.83, 22.21 y $22.01 \%$, que en términos de volumen, representaría pasar de una producción de 82.57, 193.73, 190.05 miles de t. registrada durante 2016 
a $121.23,236.75$ y 231.88 miles de toneladas. Dichos resultados concuerdan con los reportados en el periodo 1970-1998 ${ }^{(6)}$, ya que durante este término, obtuvieron una elasticidad para la oferta de carne de pollo en México en relación al cambio tecnológico, de 1.972.

La cantidad ofrecida de carne de pollo respecto al precio del producto a precios actuales y con un

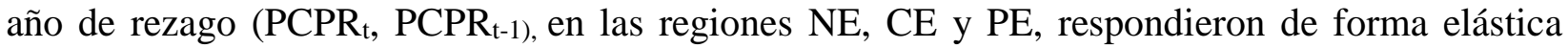
$(3.633,1.579,1.707)$, e indica que ante un incremento en una unidad porcentual en el precio de la carne de pollo en canal, la cantidad ofrecida responde en porcentaje mayor a la unidad en estas regiones; en tanto, las zonas Noroeste, Norte, Centro Occidente, Sur, Oriente, respondieron de forma inelástica $(0.002,0.282,0.813,0.094,0.162)$; esto significa que ante un incremento en una unidad porcentual del precio de la carne de pollo en canal, provoca un aumento no significativo en la cantidad ofrecida del producto en estas regiones. Estos hallazgos concuerdan con los reportados por otros investigadores ${ }^{(13,26,27)}$, quienes obtuvieron valores inelásticos de la oferta de carne de pollo en relación a su precio.

La respuesta de la oferta de carne de pollo en canal, respecto a los cambios ocurridos en el precio de la carne de cerdo, a precio actual y con dos años de rezago $\left(\mathrm{PCCR}_{\mathrm{t}}, \mathrm{PCCR}_{\mathrm{t}-2}\right)$, como un bien alternativo, fue inelástica en la mayoría de las regiones productoras, excepto en la zona NE que fue elástica. Ante este escenario, los productores de carne de pollo y cerdo en esta región, deciden que ante una mejora en el precio de la carne de cerdo; podrían estimularse para producir más cerdo y, esta decisión, afectaría la oferta de carne de pollo en este territorio; en tanto, que en el resto de las zonas el efecto no es significativo.

Por otra parte, la dinámica de la oferta de carne de pollo en canal en relación a los cambios ocurridos en el precio del alimento a precio actual y con dos años de rezago $\left(\mathrm{PAR}_{\mathrm{t}}, \mathrm{PAR}_{\mathrm{t}-2}\right)$, difiere entre las regiones. En las regiones NE y NR la magnitud de los coeficientes fueron menos inelásticos (-.0144, -0.190) que el resto de las zonas; es decir el volumen de carne de pollo en canal producido en esas regiones respondió inversamente y en mayor proporción ante variaciones en el precio del insumo.

Sin embargo, las zonas CO y PE fueron más inelásticas $(-0.008,-0.003)$ que la totalidad de las regiones, lo que mostró, que ante un incremento en una unidad porcentual en el precio del alimento $\left(\mathrm{PAR}_{\mathrm{t}}, \mathrm{PAR}_{\mathrm{t}-2}\right)$, la oferta de carne de pollo en canal en dichas zonas geográficas registró decrementos poco representativos. Cabe señalar que para el periodo $1978-1998^{(6)}$, obtuvieron para México, un coeficiente de -0.164 respecto al precio esperado del sorgo (como su principal componente del alimento para pollo). No obstante que la magnitud de la elasticidad varía entre los diversos estudios por tratarse de periodos distintos, pero permanece el carácter inelástico.

En lo que respecta a las elasticidades de demanda se encontró que la variable que mayor efecto registró sobre el consumo de carne de pollo en canal en todas las regiones fue el tamaño de la población, toda vez que su comportamiento fue elástico en todas las regiones; sin embargo, fueron 
las regiones OR, SUR y NR, las que reportaron mayores elasticidades (3.1295, 2.3610 y 2.1045); por tanto, aumentos significativos en el consumo de pollo, ante un cambio en una unidad porcentual en el tamaño de la población. Respecto de las regiones CO, NO y CE, la elasticidad fue de 2.0220, 1.9750 y 1.7868 , lo que indicó que la demanda de carne de pollo en dichas regiones se incrementó en proporción menor que en los territorios anteriores. El efecto que registró la variable población sobre el consumo de carne de pollo en las diferentes regiones de México, se explica en gran generalidad por el crecimiento medio anual de $1.22 \%$ de la población nacional durante el periodo de estudio.

En una investigación realizada en relación con el comportamiento de la oferta y demanda regional de carne de cerdo en canal en México ${ }^{(23)}$, los autores concluyeron que la cantidad demandada de carne de cerdo respondió de forma elástica en todas las regiones de México en relación al crecimiento de la población humana; estos resultados concuerdan con los obtenidos en el presente estudio, ya que la demanda de carne de pollo en canal en todas las regiones en relación al crecimiento de la población humana fue elástica.

En lo que se refiere a la elasticidad de la demanda de carne de pollo en canal respecto a su precio corriente fue inelástica en todas las zonas; sin embargo, existen divergencias en los valores de cada una de ellas. En las regiones PE, SUR y NE, los valores fueron menos inelásticos $(-0.0819,-0.1014$, -0.1037) que el resto de las mismas; ello evidenció que esta variable económica afectó en proporción menor el consumo de carne de pollo en esas regiones; lo anterior, debido a diferencias en el ingreso per cápita y precio del producto sustituto entre territorios. Dichos resultados, concuerdan con los reportados por otros investigadores ${ }^{(26-29)}$, quienes reportaron valores en relación a su precio de $-0.36,-0.4718,1.191,-0.2148,-0.1695$, cifras que resultaron ligeramente diferentes a las reportadas en esta investigación, por tratarse de periodos diferentes; sin embargo permanece el perfil inelástico.

Por otra parte, la elasticidad de la demanda en relación al producto interno bruto per cápita, registró cambios a nivel regional, mientras que en los territorios NO, OR y PE, se obtuvieron valores de $0.0741,0.1428$ y 0.1536 , otras regiones como CE, CO y NR, reportaron coeficientes superiores (0.5457, 0.3557 y 0.2844$)$, que el resto de las mismas. Los valores de magnitud mayor significaron que el consumo de carne de pollo se explicó en proporción mayor por el aumento en el PIBRP $\mathrm{t}_{\mathrm{t}}$ En México se reportó una elasticidad de 0.3347, para el periodo $1970-1998^{(6)}$, respecto al ingreso per cápita real disponible.

La elasticidad de la demanda en relación al precio de la carne de bovino, hubo variaciones a nivel territorial, mientras que en las regiones $\mathrm{NE}$, NO y NR, se obtuvieron elasticidades de 0.0817, 0.0656 y 0.0506 , las zonas SUR, PE y OR, reportaron menores magnitudes $(0.0001,0.0001$ y 0.0017), lo que indicó que el efecto causado por el incremento del precio de la carne de bovino sobre la demanda de carne de pollo en estas regiones fue mínimo. 


\section{Conclusiones e implicaciones}

El factor que más influyó en el crecimiento de la producción avícola en la mayoría de las regiones de México, fue el progreso tecnológico, definido como eficiencia alimenticia. La demanda regional de carne de pollo, fue elástica en relación al crecimiento de la población; es decir, el incremento del número de personas influyó en proporción mayor en el aumento del consumo de carne de pollo en todas las regiones. Con los resultados obtenidos, se determinó el porcentaje de afectación que ejercieron las variables explicativas en la oferta y demanda regional de carne de pollo en México.

\section{Literatura citada:}

1. Medina CJC, Rejón AMJ, Valencia, HER. Análisis de rentabilidad de la producción y venta de pollo en canal en el municipio de Acanceh, Yucatán, México. Rev Mex Agroneg 2012;16(30):909-919.

2. UNA. Unión Nacional de 2018. http://www.una.org.mx/index.php/component/content/article/15-panorama/3-avicultura. Consultado Feb 05, 2018.

3. FIRA. Fideicomisos Instituidos en Relación con la Agricultura. Panorama Agroalimentario. Dirección de Investigación y Evaluación Económica y Sectorial. Avicultura Carne. 2016. https://www.gob.mx/cms/uploads/attachment/file/200631/Panorama_Agroalimentario_Avic ultura_Carne_2016.pdf. Consultado Nov 14, 2016.

4. SIAP. Servicio de Información Agroalimentaria y Pesquera. SAGARPA. Base de datos. 2016. http://www.siap.gob.mx/index.php?option=com_wrapper\&view=wrapper\&Itemid=371 . Consultado Oct 24, 2016.

5. FIRA. Fideicomisos Instituidos en Relación con la Agricultura. Panorama Agroalimentario. Dirección de Investigación y Evaluación Económica y Sectorial. Avicultura Carne. 2015. https://www.gob.mx/cms/uploads/attachment/file/61946/Panorama_Agroalimentario_Avicul tura_Carne_2015.pdf. Consultado Ago 22, 2016.

6. Ramírez JA, García MR, García DG, Matus GJ. Un modelo de ecuaciones simultáneas para el mercado de la carne de pollo en México, 1970-1998. Agrociencia 2003;37(1):73-84.

7. INEGI. Instituto Nacional de Estadística y Geografía. Banco de Información Económica. http://www.inegi.org.mx/sistemas/bie/. Consultado Dic 29, 2017.

8. CONAPO. Consejo Nacional de población. Indicadores demográficos de México, periodo 1990-2030. https://datos.gob.mx/herramientas/indicadores-demograficos-de-mexicoperiodo-1990-2030?category=web\&tag=economia. Consultado Oct 05, 2017 
9. Téllez DR, Mora FJS, García MR, Martínez DMA. Caracterización del consumidor de carne de pollo en la zona metropolitana del Valle de México. Rev Estudios Soc 2016;48(26):193209.

10. Del Moral BLE, Ramírez GBP, Muñoz, JAR. Crecimiento regional de la producción de carne de cerdo en México 1980-2005. Análisis Económico 2008;52(23):272-290.

11. Bassols BA. Geografía económica de México. Formación de regiones económicas. $1^{\mathrm{a}}$ reimpresión, México, DF: Ed, Trillas; 1992.

12. Salvatore D. Teoría y problemas de microeconomía, México, DF: Ed, McGraw-Hill; 1997.

13. Vázquez AJMP, Martínez DMA. Elasticidades de oferta y demanda de los principales productos agropecuarios de México. Secretaría de Agricultura, Ganadería, Desarrollo Rural, Pesca y Alimentación (SAGARPA). Instituto Nacional de Investigaciones Forestales, Agrícolas y Pecuarias (INIFAP). Centro de Investigación Regional Pacífico Sur. Campo Experimental "Zacatepec”. Zacatepec, Morelos, México. Publicación especial. Num. 51; 2011.

14. Rigolin P. Global poultry director for Alltech. Global Champion of Allzyme SSF, Alltech, Inc., Lexington, Kentucky, USA. Evolución de la conversión alimenticia en pollos de engorde. 2014. http://www.wattagnet.com/articles/17830-conversion-alimenticia-1-1-para-2025-unvistazo-al-futuro-de-la-avicultura

15. Gujarati ND, Porter DC. Econometría. 5ta ed. México, DF: McGraw-Hill Interamericana; 2010.

16. SAS. Statistical Analysis System 2003. Versión 9.1.3 SAS Institute Inc., Cary, NC, USA.

17. Hall RLM, Lieberman, M. Macroeconomía. Principios y aplicaciones. 3ra ed. Thomson; 2006.

18. SAGARPA. Secretaría de Agricultura, Ganadería, Desarrollo Rural, Pesca y Alimentación. Situación actual y perspectiva de la producción de carne de pollo en México. 1998. http://www.sagarpa.gob.mx/ganaderia/Publicaciones/Lists/Estudios\%20de\%20situacin\%20a ctual\%20y\%20perspectiva/Attachments/15/sitpollo97.pdf. Consultado May 19, 2017.

19. Bachoco. https://bachoco.com.mx/el-principio-del-sabor/procesos-del-cerdo/ Consultado Oct 27, 2017.

20. Pilgrims. http://www.pilgrims.com.mx/. Consultado Oct 28, 2017.

21. Tyson (John Tyson, Chaitman). https://www.tysonfoods.com/who-we-are. Consultado Oct 27, 2017. 
22. SENASICA. Servicio Nacional de Sanidad, Inocuidad y Calidad Agroalimentaria. Unidades de Producción Avícola Registradas. 2017. https://datos.gob.mx/busca/dataset/unidades-deproduccion-avicola-registradas. Consultado Jul 10, 2017.

23. Rebollar RA, Gómez TG, Hernández MJ, Rebollar RS, González RFJ. Comportamiento de la oferta y demanda regional de carne de cerdo en canal en México, 1994-2012. Rev Mex Cienc Pecu 2014;5(4):377-392.

24. Pérez VFC, García MR, Martínez DMA, Mora FJS, Vaquera HH, González EA. Efecto de las importaciones de carne de porcino en el mercado mexicano, 1961-2007. Rev Mex Cienc Pecu 2010;1(2):115-126.

25. Klein LR. An introduction to econometrics. Prentice Hall, Englewood Cliffs-New York, USA. 1962.

26. Bhati UN. Supply and demand responses for poultry meat in Australia. Australian J Agr Econom 1987;31(3):256-265.

27. Vázquez AJMP, Martínez DMA. Estimación empírica de elasticidades de oferta y demanda. Rev Mex Cienc Agríc 2015;6(5):955-965.

28. Ramírez TJ, Martínez DMA, García MR, Hernández GA, Mora FJS. Aplicación de un sistema de demanda casi ideal (AIDS) a cortes de carnes de bovino, porcino, pollo, huevo y tortilla en el periodo 1995-2008. Rev Mex Cienc Pecu 2011;2(1):39-52.

29. González SRF. Estimación de elasticidades de demanda para la carne de res, pollo, cerdo y huevo en México, una aplicación del Sistema de Demanda Casi Ideal. [tesis doctoral]. México: Universidad Autónoma Chapingo; 2001. 